\title{
Effect of vitamin D supplementation on circulating concentrations of C-reactive protein (CRP) in adults aged $20-40$ and $64+$ years
}

\author{
A. Lucey $^{1}$, T. Hill ${ }^{1}$, S. Muldowney ${ }^{1}$, K. Seamans ${ }^{1}$, J. M. W. Wallace ${ }^{3}$, G. Horigan ${ }^{3}$, M. S. Barnes ${ }^{3}$, \\ K. D. Cashman ${ }^{1,2}$ and M. Kiely ${ }^{1}$ \\ ${ }^{1}$ Departments of Food and Nutritional Sciences, ${ }^{2}$ Medicine, University College Cork, Ireland and ${ }^{3}$ Northern Ireland Centre \\ for Food and Health, University of Ulster, Coleraine, BT52 1SA, Northern Ireland
}

C-reactive protein (CRP) is a measure of systemic inflammation; elevations in CRP concentrations are linked to increased risk of cardiovascular events ${ }^{(1)}$. Vitamin D supplementation decreased circulating CRP concentrations in adults with serum 25-hydroxyvitamin $\mathrm{D}(25(\mathrm{OH}) \mathrm{D})$ levels $<27.5 \mathrm{nmol} / \mathrm{l}^{(2)}$.

The effect of vitamin D supplementation $\left(0,5,10\right.$ and $15 \mu \mathrm{g}$ vitamin $\left.\mathrm{D}_{3} / \mathrm{d}\right)$ on circulating CRP concentrations was examined in two randomised, placebo-controlled, double-blind 22-week intervention studies in men and women aged 20-40 years $(n=213$; during winter $\left.2006 / 07^{(3)}\right)$ and $64+$ years $\left(n=209\right.$; during winter 2007/08 $\left.{ }^{(4)}\right)$ from Cork and Coleraine. Fasting serum levels of high sensitivity CRP and $25(\mathrm{OH}) \mathrm{D}$ were measured by ELISA at baseline $(\mathrm{BL})$ and endpoint $(\mathrm{EP})$.

No baseline differences in concentrations of CRP or $25(\mathrm{OH}) \mathrm{D}$ were observed between the four treatment groups. At BL, in the 20-40 year olds, linear regression analysis showed BMI to be the main determinant of CRP concentrations $(\beta=0.351 ; 95 \%$ CI $0.027,0.058$; $P<0.001)$. In the $64+$ years group, age $(\beta=0.140 ; 95 \%$ CI $0.000,0.024 ; P=0.046)$, systolic blood pressure $(\beta=0.143 ; 95 \%$ CI 0.000 , $0.007 ; P=0.041)$ and BMI $(\beta=0.139 ; 95 \%$ CI $0.000,0.026 ; P=0.042)$ were the main determinants of CRP levels. Vitamin D supplementation significantly influenced $25(\mathrm{OH}) \mathrm{D}$ concentrations ${ }^{(3,4)}$. In both age groups, $25(\mathrm{OH}) \mathrm{D}$ concentrations were not related to CRP. Using CRP concentrations at EP as the dependent variable, ANCOVA, controlling for centre, age, gender, BMI, baseline 25(OH)D (and systolic blood pressure for $64+$ years), showed no effect of treatment across the four groups. In conclusion, vitamin D supplementation had no effect on circulating CRP concentrations in apparently healthy adults aged 20-40 and 64 + years.

\begin{tabular}{|c|c|c|c|c|c|c|}
\hline & & Placebo & $5 \mu \mathrm{g} / \mathrm{d}$ & $10 \mu \mathrm{g} / \mathrm{d}$ & $15 \mu \mathrm{g} / \mathrm{d}$ & $P^{*}$ \\
\hline $20-40$ years & & $(n=56)$ & $(n=50)$ & $(n=57)$ & $(n=50)$ & \\
\hline \multirow[t]{2}{*}{ CRP (mg/l) } & BL & $1.70(0.62,3.35)$ & $2.14(0.75,4.13)$ & $1.93(0.67,4.69)$ & $1.76(0.75,3.39)$ & 0.599 \\
\hline & $\mathrm{EP}$ & $1.84(0.87,3.56)$ & $2.05(0.94,3.79)$ & $1.76(0.83,3.66)$ & $1.58(0.51,4.39)$ & 0.576 \\
\hline \multirow[t]{2}{*}{$25(\mathrm{OH}) \mathrm{D}^{3}(\mathrm{nmol} / \mathrm{l})$} & BL & $65.7(57.2,95.5)$ & $60.0(50.1,88.9)$ & $72.2(56.7,93.1)$ & $77.2(59.4,89.7)$ & 0.449 \\
\hline & $\mathrm{EP}$ & $37.4(30.9,48.1)^{\mathrm{a}}$ & $50.5(44.8,60.6)^{\mathrm{b}}$ & $59.6(49.4,69.5)^{\mathrm{c}}$ & $69.8(59.4,84.7)^{\mathrm{d}}$ & $<0.001$ \\
\hline $64+$ years & & $(n=54)$ & $(n=48)$ & $(n=56)$ & $(n=51)$ & \\
\hline \multirow[t]{2}{*}{$C R P(m g / L)$} & BL & $2.63(1.65,3.81)$ & $2.37(1.03,4.39)$ & $2.68(1.60,5.13)$ & $2.93(1.75,5.08)$ & 0.808 \\
\hline & EP & $2.43(1.20,4.87)$ & $2.22(0.96,3.50)$ & $1.95(1.18,4.11)$ & $3.03(1.21,4.85)$ & 0.540 \\
\hline \multirow[t]{2}{*}{$25(\mathrm{OH}) \mathrm{D}^{4}(\mathrm{nmol} / \mathrm{l})$} & BL & $59.5(42.7,78.6)$ & $51.8(40.3,72.6)$ & $53.3(41.9,72.3)$ & $52.8(38.5,67.7)$ & 0.715 \\
\hline & $\mathrm{EP}$ & $41.0(27.8,55.9)^{\mathrm{a}}$ & $52.1(45.5,68.7)^{\mathrm{b}}$ & $69.2(58.0,81.2)^{\mathrm{c}}$ & $71.1(61.4,87.3)^{\mathrm{d}}$ & $<0.001$ \\
\hline
\end{tabular}

Values presented as median (IQR). Values with different superscript letters are significantly different, $P<0.001$. *ANCOVA.

We acknowledge the UK Food Standards Agency and the Irish Department of Agriculture, Food and Fisheries through the Food Institutional Research Measure for their support.

1. Ridker PM \& Silvertown JD (2008) J Periodontal. 79, 1544-1551.

2. Timms PM, Mannan N, Hitman GA et al. (2002) Q J Med 95, 787-796.

3. Cashman KD, Hill TR, Lucey AJ et al. (2008) Am J Clin Nutr 88, 1535-1542.

4. Cashman KD, Wallace JMW, Horigan G et al. (2009) Am J Clin Nutr 89, 1-9. 